\title{
Emodin improves the cardiac function in the rats with chronic heart failure through regulation of the miR-26b-5p/PTEN pathway
}

\author{
Zuncai Gu, Shuhua Zhang, Siyuan Zhao, Ying Cui, Liming Sun
}

Department of Cardiovasology, The Second People's Hospital of Lianyungang, Jiangsu 222023, China

Submitted: 13 August 2019

Accepted: 19 November 2019

Arch Med Sci

DOI: https://doi.org/10.5114/aoms.2020.95464

Copyright @ 2020 Termedia \& Banach

\section{Abstract}

Introduction: Chronic heart failure (CHF) is a leading cause of deaths induced by cardiovascular disease. This study aimed to investigate the protective effects of emodin in CHF rats and explore the related mechanisms.

Material and methods: A total of 56 Wistar rats were used to construct $\mathrm{CHF}$ model using the coronary artery ligation. The effects of emodin on cardiac function and inflammation were analyzed in the CHF rats. Expression of miR-26b-5p in the CHF model before and after emodin treatment was estimated by quantitative real-time polymerase chain reaction. The effects of miR-26b-5p on cardiac function and inflammation were also assessed, and its target gene was predicted and confirmed in rat cardiomyocyte H9c2. Results: Emodin treatment could significant improve the cardiac function and inflammation evidenced by the increased increased ejection fraction (EF), fractional shortening (FS), left ventricular systolic pressure (LVSP) and maximum of the first differentiation of left ventricular pressure $\left(+L V d P / d t_{\max }\right)$ and decreased atrial natriuretic peptide (ANP), brain natriuretic peptide (BNP), left ventricular end diastolic pressure (LVEDP), interleukin (IL)-6, tumor necrosis factor $\alpha$ (TNF- $\alpha$ ) levels. Expression of miR-26b-5p was downregulated in the CHF rats (CHF $0.442 \pm 0.131$ vs. Sham $1.044 \pm 0.160$ ), and this suppressive effect was rescued by emodin (Emodin $0.902 \pm 0.132$ vs. CHF $0.442 \pm 0.131$ ). The overexpression of miR-26b-5p in CHF rats led to improved cardiac function and inflammatory response. In addition, the emodin-induced increased EF, FS, LVSP and $+\mathrm{LV} \mathrm{dP/dt} \mathrm{m}_{\max }$ and decreased ANP, BNP, LVEDP, IL- 6 and TNF- $\alpha$ were all abrogated by the knockdown of miR-26b-5p. The target prediction results revealed that PTEN was a target gene of miR-26b-5p in $\mathrm{H} 9 \mathrm{c} 2$ cells.

Conclusions: All the results indicated that emodin serves a protective role in CHF via regulation of the miR-26b-5p/PTEN pathway. Emodin may be an effective therapeutic agent for $\mathrm{CHF}$ treatment.

Key words: emodin, chronic heart failure, MiR-26b-5p, PTEN, inflammation.

\section{Introduction}

Cardiovascular disease is considered one of the most serious healthy burdens and responsible for a large part of global mortality [1]. The incidence rate of acute myocardial infarction has declined in recent years due to the advanced therapeutic regimens, but the cardiovascular diseaseinduced mortality has increased mainly because of chronic heart failure (CHF) [2]. Chronic heart failure is an abnormality of cardiac structure or

\author{
Corresponding author: \\ Zuncai Gu \\ Department of Cardiovasology \\ The Second People's Hospital \\ of Lianyungang \\ No. 161, Xingfu South Road \\ Lianyungang, Jiangsu 222023 \\ China \\ Phone: 86-0518-85775071 \\ Fax: 86-0518-85214221 \\ E-mail: \\ guzuncai2075@126.com
}


function that results in the heart fails to pump sufficiently to maintain blood flow to meet the body needs [3]. Patients suffering from CHF have some common clinical symptoms, including excessive tiredness, shortness of breath and leg swelling, leading to the decreased quality of life and increased economic burden on health care [4]. It is reported that some risk factors are closely related with the initiation and development of CHF, such as myocardial infarction, myocarditis, high blood pressure, abnormal inflammation, alcohol abuse and myocardial disfunction [5]. Atrial natriuretic peptide (ANP) and brain natriuretic peptide (BNP) belong to the natriuretic peptides and are closely associated with the development of CHF [6]. Although a great progress has been achieved in the management of $\mathrm{CHF}$, the outcomes of the patients remain dismal [7]. Thus, novel therapeutic strategies are urgently needed.

Emodin (1, 3, 8-trihydroxy-6-methylanthraquinoe) is an active constituent of Rheum officinale and POlygonum cuspidatum $[8,9]$. Abundant studies indicate that emodin has obviously anti-inflammatory effects in various diseases and abnormal conditions by inhibition of pro-inflammatory cytokines and suppression of $\mathrm{T}$ lymphocyte proliferation $[10,11]$. Recent research found that emodin serves protective role against some cardiovascular diseases. For example, Song et al. demonstrated that emodin could ameliorate the experimental autoimmune myocarditis in rats [12]. Wu and his colleagues also reported the protective effects of emodin in acute myocardial infarction, which performed by regulation of inflammation [13]. However, the role of emodin in $\mathrm{CHF}$ treatment has been rarely investigated.

Emerging studies have focused on the role of microRNAs (miRNAs) in the molecular mechanisms underlying the protective effects of emodin. In lipopolysaccharide-induced injury $\mathrm{H} 9 \mathrm{c} 2$ cells, emodin was proved to improve the myocarditis by regulating miR-223 and the downstream inflammatory cytokines [14]. In myocardial infarction cell model, emodin could ameliorate the hypoxia-induced injury through upregulation of miR-138 [15]. A significant low expression of miR-26b-5p has been reported in the study by Eline et al. in the patients with CHF compared with healthy individuals [16] In addition, miR-26b has also been found to improve myocardial infarction by relieving inflammatory response in mice [17]. However, the biological function of miR-26b in the development of $\mathrm{CHF}$ and its relationship with emodin remain unclear.

In this study, we sought to investigate the protective role of emodin and explore the underlying mechanisms by examining its regulatory effects on miR-26b-5p expression and inflammation in the rats with $\mathrm{CHF}$

\section{Material and methods}

\section{Construction of rat model}

The protocols for the animal experiments were approved by the local committee of The Second People's Hospital of Lianyungang, and all the rats were treated following the Guide for the Care and Use of Laboratory Animals of the Institute for Laboratory Animal Research. The study was conducted in accordance with the Basic \& Clinical Pharmacology \& Toxicology policy for experimental and clinical studies [18]. A total of 64 10-weeksold Wistar rats with body weight of $250 \pm 30 \mathrm{~g}$ were obtained from Shanghai Laboratory Animal Center (Shanghai, China). All the rats were housed in a standard environment with free access to water and food and a $12 \mathrm{~h}$ light/dark cycle at $25^{\circ} \mathrm{C}$. The rats were randomly grouped into 8 groups with the grouping details listed in Table I. Chronic heart failure animal model was constructed by coronary artery ligation as previously described. The rats were randomly grouped into two groups: sham group and CHF model group, and were anesthetized with $50 \mathrm{mg} / \mathrm{kg}$ of sodium pentobarbital.

Table I. Treatment for different animal groups

\begin{tabular}{|c|c|c|}
\hline Groups & Number & Treatment \\
\hline Sham & 8 & Sham surgery without ligation of coronary arteries \\
\hline $\mathrm{CHF}$ & 8 & Ligation of left coronary artery near its branch point from aorta \\
\hline NS & 8 & $50 \mathrm{mg} / \mathrm{kg} /$ day normal saline by intragastric administration for 3 weeks in CHF rats \\
\hline Emodin & 8 & $50 \mathrm{mg} / \mathrm{kg} /$ day emodin by intragastric administration for 3 weeks in CHF rats \\
\hline Mimic NC & 8 & Caudal vein injection with mimic NC in CHF rats \\
\hline miR-26b-5p mimic & 8 & Caudal vein injection with miR-26b-5p mimic NC in CHF rats \\
\hline Emodin + inhibitor NC & 8 & $\begin{array}{l}\text { Caudal vein injection with inhibitor } \mathrm{NC} \text { and intragastric administration } \\
\text { of } 50 \mathrm{mg} / \mathrm{kg} \text { emodin in } \mathrm{CHF} \text { rats }\end{array}$ \\
\hline $\begin{array}{l}\text { Emodin }+\operatorname{miR}-26 b-5 p \\
\text { inhibitor }\end{array}$ & 8 & $\begin{array}{c}\text { Caudal vein injection with } \mathrm{miR}-26 \mathrm{~b}-5 \mathrm{p} \text { inhibitor and intragastric administration } \\
\text { of } 50 \mathrm{mg} / \mathrm{kg} \text { emodin in CHF rats }\end{array}$ \\
\hline
\end{tabular}


In the CHF group, a 6-0 suture was used to ligate the left coronary artery near its branch point from aorta, between the left atrium and the pulmonary artery outflow tract. In the sham group, the rats were treated the same as the CHF group without the ligation of their coronary arteries. After the surgery, all the rats received penicillin for 3 days to avoid infection. To regulate the expression of miR$26 b-5 p$, the rats in the CHF group received caudal vein injection with miR-26b-5p mimic, miR-26b-5p inhibitor, mimic negative control (mimic NC) or inhibitor negative control (inhibitor NC) as the previously described [19]. All the vectors were synthesized by GenePharma (Shanghai, China).

\section{Emodin treatment}

The CHF model rats were divided into emodin group and normal saline (NS) group. The rats in the emodin group received $50 \mathrm{mg} / \mathrm{kg}$ emodin (ref: E7881, purity $\geq 90 \%$; Sigma, Aldrich, USA) every day for 3 weeks by intragastric administration, while the rats in the NS group were treated with NS using the same dosage.

\section{Determination of cardiac function}

To measure the cardiac function of rats, the major indicators obtained from echocardiography and hemodynamic test were estimated. The rats were anesthetized using sodium pentobarbital under the electrocardiograph monitoring. The echocardiography was conducted using a PST 64A sector scanner (8-MHz probe), and the ejection fraction (EF) and fractional shortening (FS) were calculated. For the hemodynamic test, a catheter filled with heparin was inserted into the left ventricle of the rats to connect the pressure transducer and the carrier amplifier. The left ventricular systolic pressure (LVSP) and left ventricular end diastolic pressure (LVEDP) were recorded, and the maximum of the first differentiation of left ventricular pressure $\left(+\mathrm{LV} d P / \mathrm{dt}_{\text {max }}\right)$ was calculated.

\section{Enzyme-linked immune sorbent assay (ELISA)}

The expression levels of ANP and BNP and proinflammatory cytokines, including IL-1 $\beta, I L-6$ and TNF- $\alpha$, in the serum of the rats were measured by ELISA. The concentration of proteins was evaluated by a BCA assay kit (Thermo Fisher Scientific, Waltham, MA, USA), and the protein levels of ANP, BNP, IL- 6 and TNF- $\alpha$ were examined using an ELISA kit (Invitrogen, Carlsbad, CA, USA) according to the manufacturers' instruction. The absorbance at $450 \mathrm{~nm}$ was measured to calculate the final protein levels.

\section{Cell culture and transfection}

Rat cardiomyocyte cell line $\mathrm{H} 9 \mathrm{c} 2$ was purchased from the Cell Bank of the Chinese Academy of Sci- ence (Shanghai, China). The cells were cultured in Dulbecco's modified Eagle's medium (DMEM; Thermo Fisher Scientific, Waltham, MA, USA) added with $10 \%$ fetal bovine serum (FBS; Thermo Fisher Scientific, Waltham, MA, USA) at $37^{\circ} \mathrm{C}$ in a humidified atmosphere with $5 \% \mathrm{CO}_{2}$. For cell transfection, miR-26b-5p mimic or mimic NC were transfected into the cells by Lipofectamine 2000 (Invitrogen, Carlsbad, (A, USA) following the manufacturers' instruction. The cells after $48 \mathrm{~h}$ of transfection were used for the verification of target gene of miR-26b-5p.

\section{RNA extraction and quantitative real-time polymerase chain reaction ( $\mathrm{QRT} T-\mathrm{PCR}$ )}

Total RNA was extracted from the heart tissues by TRIzol reagent (Invitrogen, Carlsbad, CA, USA), and examined using a NanoDrop 2000 (Thermo Fisher Scientific, Waltham, MA, USA) regarding the purity and concentration of the RNA. An ABI miRNA reverse transcription kit (Applied Biosystem, Foster City, USA) was used to synthesize cDNA from the RNA. To analyze the expression value of miR-26b-5p, qPCR was performed using a Power SYBR green kit (Thermo Fisher Scientific, Waltham, MA, USA) and an ABI 7500 Real-Time PCR System (Applied Biosystem, Foster City, USA). U6 was used as an endogenous control gene. The final relative expression of miR-26b-5p was calculated by $2^{-\Delta \Delta c t}$ method and normalized to U6.

\section{Luciferase reporter assay}

This study preliminarily analyzed the target gene of miR-26b-5p. Phosphatase and tensin homolog (PTEN) was predicted as a potential target gene of miR-26b-5p using TargetScan (http://www. targetscan.org/vert_72/). Thus, luciferase reporter assay was carried out to confirm the target gene. The wild-type (WT) 3'-untranslated region (3'-UTR) or mutant type (MT) 3'-UTR of PTEN were integrated into the firefly luciferase reporter vectors with Rnilla luciferase (Promega, Fitchburg, WI, USA). The H9c2 cells were seeded into 24 well plates and co-transfected with the recombinant vector and miR-26b-5p mimic, miR-26b-5p inhibitor, mimic NC or inhibitor NC. The luciferase activity was detected after $48 \mathrm{~h}$ of transfection using a SecrePair Dual-Luciferase Reporter System (Promega).

\section{Statistical analysis}

SPSS statistical software (SPSS Inc., Chicago, IL) and GraphPad Prism software (GraphPad Software, Inc, USA) were used to perform all the statistical analyses, and the data were expressed as mean \pm SD. Student's t test and one-way ANOVA were used to compare the data compliance with normal distribution between groups. A $p$ value of less than 0.05 was considered statistically significant. 


\section{Results}

Emodin improves the cardiac function of the chronic heart failure rats

In this study, the CHF rat model was constructed by coronary artery ligation. The cardiac function of the rats was evaluated using the indicators shown in Table II. The results revealed that the levels of ANP and BNP and LVEDP were increased, while the EF, FS, LVSP and $+\mathrm{LV} \mathrm{dP/dt}$ max $_{\text {mere decreased }}$ in the $\mathrm{CHF}$ rats compared with the sham group (all $p<0.05)$, indicating that the CHF model was successfully obtained. After the administration of emodin, the decreased EF, FS, LVSP and $+\mathrm{LV} \mathrm{dP/dt}{ }_{\text {max }}$ and the increased ANP and BNP and LVEDP induced by CHF were all rescued by the treatment of emodin (all $p<0.05$ ).

\section{Emodin suppresses the inflammation of the chronic heart failure rats}

Considering the pivotal role of inflammation in the development of $\mathrm{CHF}$, the serum concentrations of IL- 6 and TNF- $\alpha$ were measured. As expected, the levels of IL- 6 and TNF- $\alpha$ were both significantly upregulated after the CHF modeling (all $p<0.05$ ). Notably, the increases in IL-6 and
TNF- $\alpha$ levels by CHF were abrogated by the treatment of emodin (all $p<0.05$, Figure 1 ).

\section{Expression of miR-26b-5p in the chronic heart failure rats before and after emodin treatment}

It is reported that the expression of miR-26b-5p was decreased in CHF. In consistent with the previous result, we also found the downregulated expression of miR-26b-5p in the CHF rats compared with the sham group $(p<0.05)$. Interestingly, we observed that the decreased expression of miR$26 \mathrm{~b}-5 \mathrm{p}$ in the CHF model was remarkably elevated by the treatment of emodin $(p<0.05$, Figure 2$)$.

\section{Overexpression of miR-26b-5p improves the cardiac function and inflammation of the chronic heart failure rats}

Given the decreased expression of miR-26b-5p in CHF rats, we examined its effects on cardiac function and inflammation. The expression of miR-26b-5p was upregulated by the animal transduction with miR-26b-5p ( $p<0.01$, Figure $3 \mathrm{~A})$. As shown in Table III, EF, FS, LVSP and $+\mathrm{LV} \mathrm{dP} / \mathrm{dt}_{\max }$ in the $\mathrm{CHF}$ model were promoted by the overexpres-

Table II. Effects of emodin on cardiac function of chronic heart failure rats

\begin{tabular}{|lcccc|}
\hline Parameters & Sham $(n=8)$ & CHF $(n=8)$ & NS $(n=8)$ & Emodin $(n=8)$ \\
\hline ANP $[\mathrm{ng} / \mathrm{l}]$ & $38.2 \pm 5.5$ & $77.6 \pm 4.9^{* *}$ & $78.8 \pm 4.7^{* *}$ & $43.2 \pm 4.1^{\# \#}$ \\
\hline BNP $[\mathrm{ng} / \mathrm{l}]$ & $66.4 \pm 5.1$ & $129.2 \pm 10.2^{* *}$ & $131.8 \pm 9.3^{* *}$ & $71.6 \pm 6.2^{\# \#}$ \\
\hline EF $(\%)$ & $80.4 \pm 7.1$ & $61.4 \pm 5.4^{*}$ & $59.6 \pm 4.0^{*}$ & $77.8 \pm 6.0^{\#}$ \\
\hline FS $(\%)$ & $52.2 \pm 5.0$ & $30.8 \pm 4.8^{*}$ & $28.8 \pm 5.3^{*}$ & $48.8 \pm 5.3^{\#}$ \\
\hline LVSP $[\mathrm{mm} \mathrm{Hg}]$ & $104.4 \pm 20.8$ & $64.8 \pm 10.4^{*}$ & $62.6 \pm 9.5^{*}$ & $85.7 \pm 10.5^{\#}$ \\
\hline LVEDP $[\mathrm{mm} \mathrm{Hg}]$ & $2.3 \pm 1.1$ & $21.6 \pm 4.8^{* *}$ & $20.7 \pm 4.9^{* *}$ & $5.5 \pm 2.4^{\# \#}$ \\
\hline+ LV dp/dt & $5466.0 \pm 292.7$ & $3248.4 \pm 281.2^{*}$ & $3128.2 \pm 274.6^{*}$ & $4565.4 \pm 360.2^{\#}$ \\
\hline
\end{tabular}

CHF-chronic heart failure, ANP-atrial natriuretic peptide, BNP-brain natriuretic peptide, EF-ejection fraction, FS - fractional shortening, $L V S P$ - left ventricular systolic pressure, LVEDP - left ventricular systolic pressure, $+L V d P / d t_{\text {max }}$ - maximum of the first differentiation of left ventricular pressure. ${ }^{*} P<0.05,{ }^{* *} p<0.01$ vs. sham group, ${ }^{*} p<0.05,{ }^{\# \#} p<0.01$ vs. CHF group.

A

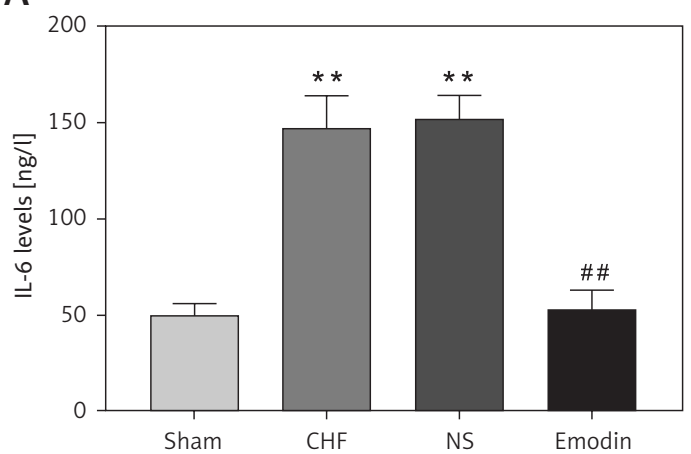

B

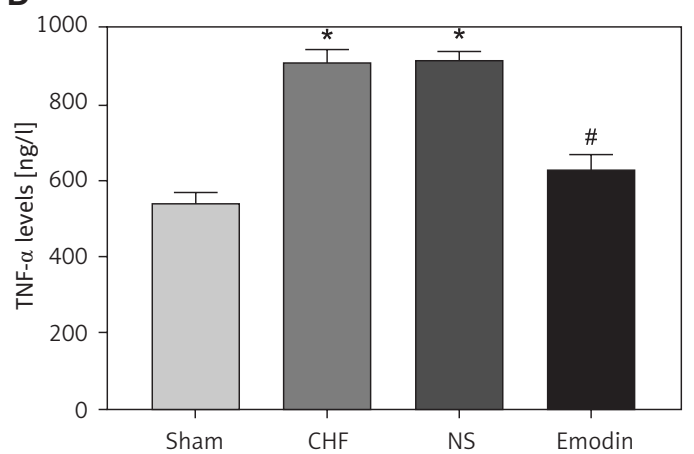

Figure 1. Proinflammatory cytokine levels in chronic heart failure (CHF) rats before and after treatment with emodin. A - Emodin could reduce the CHF-induced increased IL- 6 concentration. B - The elevated TNF- $\alpha$ level induced by CHF was decreased by emodin. Each group included 8 animals. ${ }^{*} P<0.05,{ }^{* *} p<0.01$ vs. sham group; ${ }^{*} p<0.05$, $\#$ \# $p<0.01$ vs. CHF group 
sion of miR-26b-5p, while ANP and BNP levels and LVEDP were suppressed by the upregulation of miR-26b-5p (all $p<0.05$ ). For the examination of inflammatory response, we found that the overexpression of miR-26b-5p in the CHF rats resulted in significantly reduced IL- 6 and TNF- $\alpha$ concentrations (all $p<0.05$, Figure $3 \mathrm{~B}$ and $3 \mathrm{C}$ ).

\section{MiR-26b-5p is involved in the protective effects of emodin in the chronic heart failure rats}

Since the regulatory effect of emodin on the expression of miR-26b-5p, we suspected that miR$26 b-5 p$ might be involved in the protective effects of emodin against CHF. Thus, the miR-26b-5p inhibitor was adopted to suppress the expression of miR-26b-5p in the CHF rats treated with emodin. The results in Figure $4 \mathrm{~A}$ revealed that the increased

\section{A}

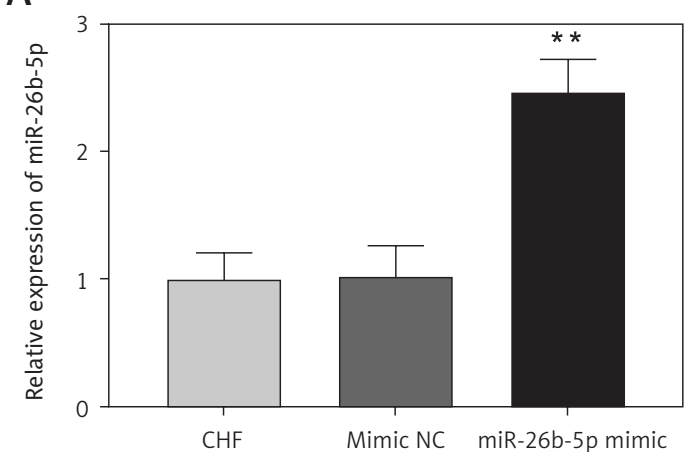

C

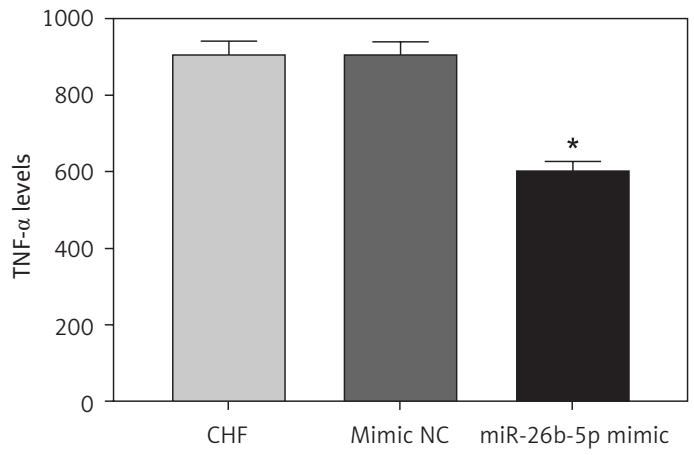

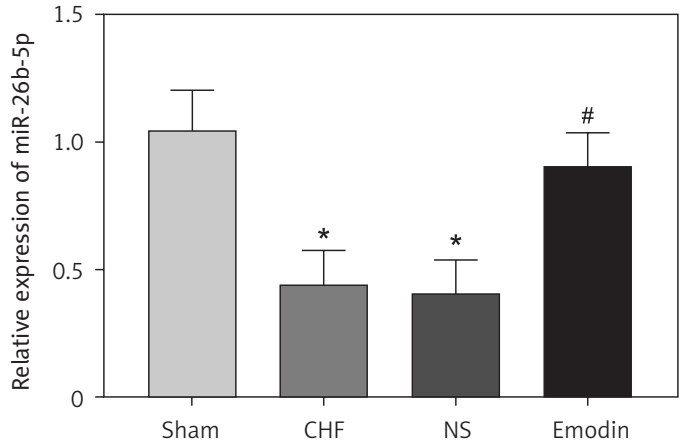

Figure 2. Expression of miR-26b-5p in chronic heart failure (CHF) rats before and after emodin administration. Expression of miR-26b-5p was upregulated in CHF, but was suppressed after treatment with emodin. Each group included 8 animals. ${ }^{\star} P<0.05$ vs. sham group; ${ }^{*} p<0.05$ vs. CHF group

B

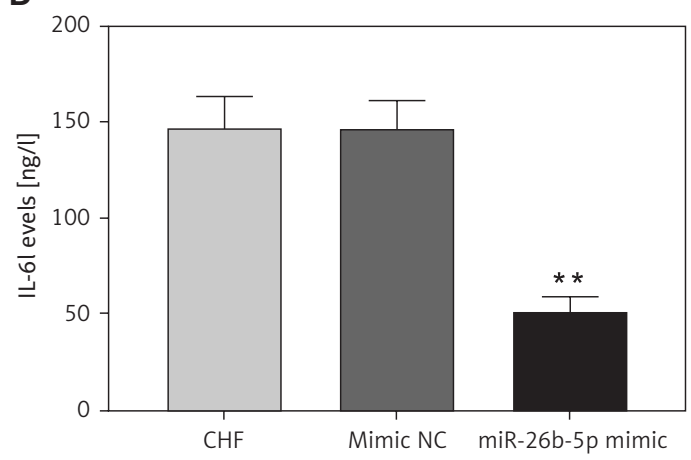

Figure 3. Effects of miR-26b-5p on inflammation in chronic heart failure (CHF) rats. A - Expression of miR-26b-5p was upregulated by miR-26b-5p mimic. B, C - Upregulation of miR-26b-5p in CHF rats resulted in decreased IL- 6 and TNF- $\alpha$ levels. Each group included 8 animals. ${ }^{*} P<0.05,{ }^{* *} p<0.01$ vs. CHF group

Table III. Effects of miR-26b-5p on the cardiac function in chronic heart failure rats

\begin{tabular}{|lccc|}
\hline Parameters & CHF $(n=8)$ & Mimic NC $(n=8)$ & miR-26b-5p mimic $(n=8)$ \\
\hline ANP $[\mathrm{ng} / \mathrm{l}]$ & $77.6 \pm 4.9^{* *}$ & $75.6 \pm 5.7$ & $47.2 \pm 3.4^{* *}$ \\
\hline BNP $[\mathrm{ng} / \mathrm{l}]$ & $129.2 \pm 10.2^{* *}$ & $130.6 \pm 9.1$ & $69.0 \pm 5.6^{* *}$ \\
\hline EF $(\%)$ & $61.4 \pm 5.4^{*}$ & $59.0 \pm 5.1$ & $75.0 \pm 5.4^{*}$ \\
\hline FS $(\%)$ & $30.8 \pm 4.8^{*}$ & $29.0 \pm 5.1$ & $48.4 \pm 5.2^{*}$ \\
\hline LVSP $[\mathrm{mm} \mathrm{Hg}]$ & $64.8 \pm 10.4^{*}$ & $64.8 \pm 12.4$ & $92.3 \pm 18.5^{*}$ \\
\hline LVEDP $[\mathrm{mm} \mathrm{Hg]}$ & $21.6 \pm 4.8^{* *}$ & $21.5 \pm 5.4$ & $5.9 \pm 2.3^{* *}$ \\
\hline+ LV dp/dt & $3248.4 \pm 281.2^{*}$ & $3156.4 \pm 262.5$ & $4827.2 \pm 338.4^{*}$ \\
\hline
\end{tabular}

CHF-chronic heart failure, ANP - atrial natriuretic peptide, BNP-brain natriuretic peptide, EF-ejection fraction, FS - fractional shortening,

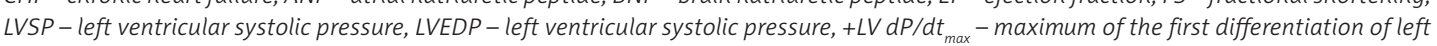
ventricular pressure. ${ }^{*} P<0.05,{ }^{* *} p<0.01$ vs. CHF group. 
A

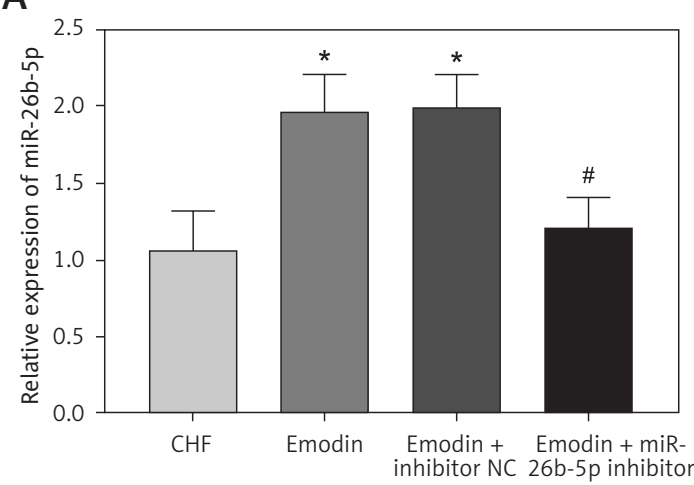

C

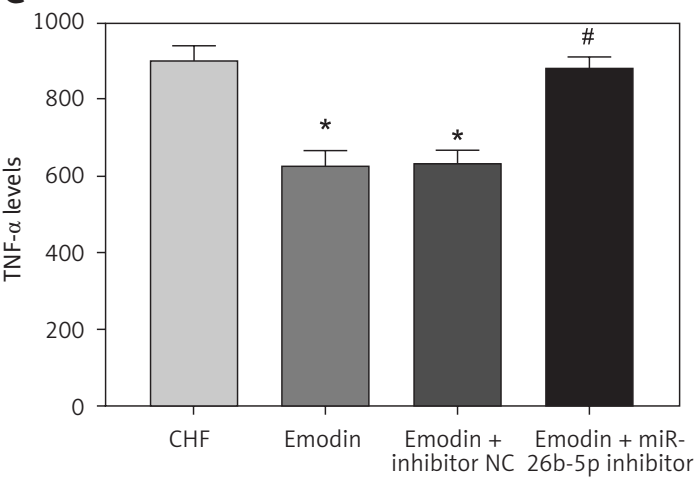

expression of miR-26b-5p induced by emodin was successfully reduced by miR-26b-5p inhibitor $(p<0.05)$. From Table IV, the emodin-induced increased EF, FS, LVSP and $+\mathrm{LV} \mathrm{dP/dt}$ max and the decreased ANP, BNP levels and LVEDP were all abrogated by the knockdown of miR-26b-5p (all $p<0.05$ ). In addition, the reduction of miR-26b-5p could enhance the emodin-induced downregulation in IL-6 and TNF- $\alpha$ levels (all $p<0.05$, Figure 4 B and 4 C).

Phosphatase and tensin homolog acts as a target for miR-26b-5p in cardiomyocytes

In the 3'-UTR of PTEN, we found the complementary sequences of miR-26b-5p (Figure $5 \mathrm{~A}$ ). Thus, we used luciferase reporter assay to confirm the inter-
B

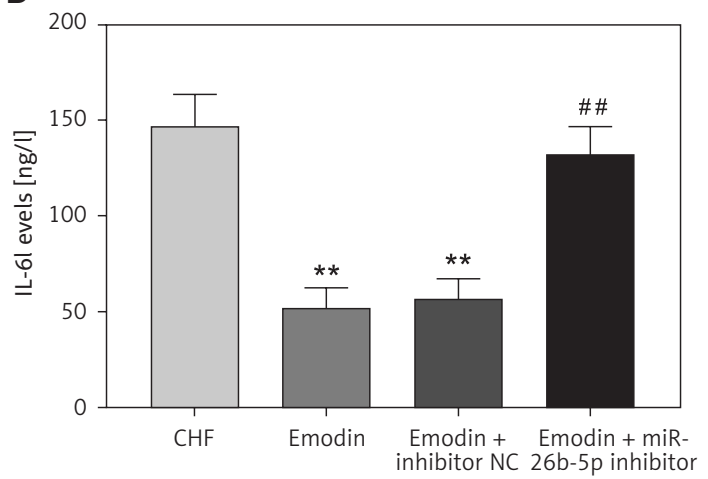

Figure 4. Inhibition of miR-26b-5p rescued the effects of emodin on inflammation in chronic heart failure (CHF) rats. A - Expression of miR-26b$5 p$ was downregulated by miR-26b-5p inhibitor. B, C - The reduced levels of IL- 6 and TNF- $\alpha$ induced by emodin were elevated by knockdown of miR26b-5p. Each group included 8 animals. ${ }^{\star} P<0.05$, ${ }^{* *} p<0.01$ vs. CHF group; ${ }^{*} p<0.05,{ }^{\# \#} p<0.01$ vs. Emodin group

action between miR-26b-5p and PTEN in the rat cardiomyocyte cell line $\mathrm{H} 9 \mathrm{c} 2$. As shown in Figure $5 \mathrm{~B}$, the expression of miR-26b-5p in the cells was upregulated by the miR-26b-5p mimic, but was downregulated by the miR-26b-5p inhibitor (all $p<0.01$ ). The luciferase activity results in the WT 3'-UTR of PTEN group were significantly inhibited by the overexpression of miR-26b-5p, whereas were promoted by the knockdown of miR-26b-5p (all $p<0.05$, Figure $5 \mathrm{C}$ ). However, no effect of miR-26b-5p was observed on the luciferase activity in the MT group.

\section{Discussion}

Traditional Chinese medicine has attracted increasing attention in the treatment of cardiovascu-

Table IV. miR-26b-5p acted as a mediator in the protective effects of emodin on cardiac function in chronic heart failure rats

\begin{tabular}{|lcccc|}
\hline Parameters & $\begin{array}{c}\text { CHF } \\
(n=8)\end{array}$ & $\begin{array}{c}\text { Emodin } \\
(n=8)\end{array}$ & $\begin{array}{c}\text { Emodin }+ \text { inhibitor } \\
\text { NC }(n=8)\end{array}$ & $\begin{array}{c}\text { miR-26b-5p inhibi- } \\
\text { tor }(n=8)\end{array}$ \\
\hline ANP [ng/l] & $77.6 \pm 4.9$ & $43.2 \pm 4.1^{* *}$ & $43.0 \pm 5.3^{* *}$ & $74.4 \pm 4.8^{\#}$ \\
\hline BNP [ng/l] & $129.2 \pm 10.2$ & $71.6 \pm 6.2^{* *}$ & $71.4 \pm 5.9^{* *}$ & $120.2 \pm 7.2^{\# \#}$ \\
\hline EF $(\%)$ & $61.4 \pm 5.4$ & $77.8 \pm 6.0^{*}$ & $76.8 \pm 4.1^{*}$ & $63.8 \pm 5.4^{\#}$ \\
\hline FS $(\%)$ & $30.8 \pm 4.8$ & $48.8 \pm 5.3^{*}$ & $48.6 \pm 5.2^{*}$ & $36.0 \pm 4.7^{\#}$ \\
\hline LVSP [mm Hg] & $64.8 \pm 10.4$ & $85.7 \pm 10.5^{*}$ & $85.7 \pm 10.9^{*}$ & $69.1 \pm 11.7^{\#}$ \\
\hline LVEDP [mm Hg] & $21.6 \pm 4.8$ & $5.5 \pm 2.4^{* *}$ & $6.1 \pm 2.8^{* *}$ & $18.5 \pm 4.2^{\# \#}$ \\
\hline$+\mathrm{LV} \mathrm{dp} / \mathrm{dt} t_{\max }[\mathrm{mm} \mathrm{Hg} / \mathrm{s}]$ & $3248.4 \pm 281.2$ & $4565.4 \pm 360.2^{*}$ & $4632.0 \pm 413.2^{*}$ & $3461.0 \pm 313.6^{\#}$ \\
\hline
\end{tabular}

CHF-chronic heart failure, ANP-atrial natriuretic peptide, BNP-brain natriuretic peptide, EF-ejection fraction, FS - fractional shortening, $L V S P$ - left ventricular systolic pressure, LVEDP - left ventricular systolic pressure, $+L V d P / d t_{\max }-$ maximum of the first differentiation of left ventricular pressure. ${ }^{*} P<0.05,{ }^{* *} p<0.01$ vs. CHF group; ${ }^{*} p<0.05,{ }^{\# \#} p<0.01$ vs. Emodin group. 
A

Position 1261-1268 of PTEN 3'-UTR

ron-miR-26b-5p

Mutant type PTEN 3'-UTR

B

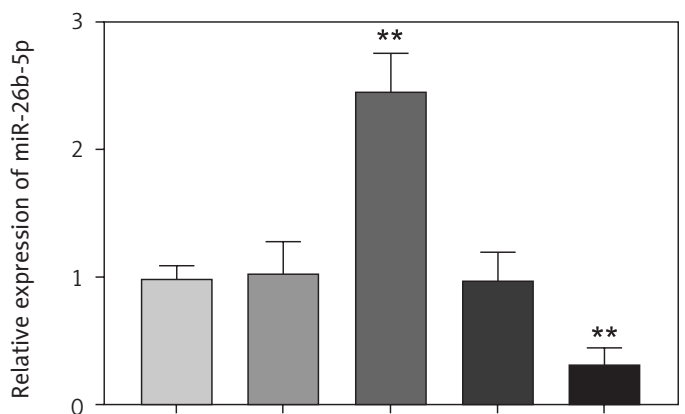

$\square$ Untreated

$\square$ mir-26b-5p mimic

$\square$ mir-26b-5p inhibitor

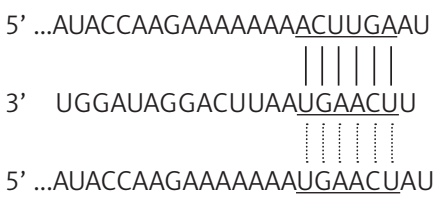

C

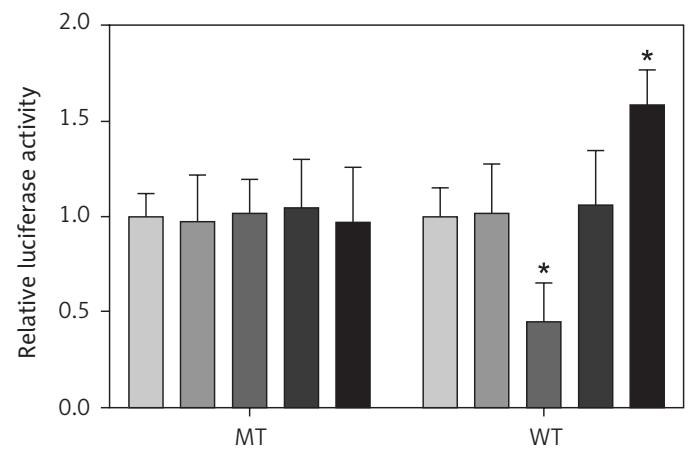

Mimic NC

- Inhibitor NC

Figure 5. PTEN served as a target gene of miR-26b-3p in H9c2 cells. A - A complementary sequence of miR-26b-5p was predicted in the $3^{\prime}$-UTR of PTEN. B - Expression of miR-26b-5p was upregulated by miR-26b-5p mimic, but was downregulated by miR-26b-5p inhibitor in H9c2 cells. C - Overexpression of miR-26b-5p suppressed the luciferase activity, but the downregulation of miR-26b-5p promoted the luciferase activity in the WT group. ${ }^{\star} P<0.05$, ${ }^{*} p<0.01$ vs. untreated group

lar diseases, especially in atherosclerotic cardiovascular disease and CHF [20, 21]. For example, Xian et al. performed a multicenter randomized double-blind placebo-controlled trial to investigate the effect of yangxinkang tablets on $\mathrm{CHF}$, and found that yangxinkang tablets could improve CHF regarding the cardiac function and quality of life in CHF patients [22]. Another study by Li et al. also prove the clinical efficacy of qili qiangxin capsules in the patients with CHF [23]. As an effective constituent of Rheum officinale, emodin has been investigated in some human disease, especially in cardiovascular diseases. In atherosclerosis, emodin was demonstrated to suppress the inflammatory response and acted an anti-atherosclerotic role [24]. In myocardial infarction, emodin could ameliorate this disease by inhibiting the inflammation and cell apoptosis [13]. In myocarditis, emodin was also involved in the regulation of proinflammatory cytokines IL-1 $\beta$ and TNF- $\alpha$, leading to inhibited inflammation in the rats with myocarditis, and thereby improved this disease [12]. However, rarely report regarding the role of emodin in the development of CHF. In the present study, we constructed the CHF model using coronary artery ligation in rats, and found that the cardiac function was impaired after the modeling. After the treatment of emodin, the impaired cardiac function was improved, which was indicated by the increased EF, FS, LVSP and $+\mathrm{LV} \mathrm{dP/dt}$ max and the decreased ANP and BNP levels and LVEDP. Thus, we considered that emodin had significantly protective effects against $\mathrm{CHF}$ in rats.
It is known that inflammation plays critical role during the development of various diseases [2528], especially in cardiovascular diseases, such as myocardial infarction [29], myocarditis [30] and also CHF [31]. Emodin has been determined as an important regulator of inflammation. It could suppress the inflammation in various diseases, such as jejunum injury [32] and acute lung injury and acute respiratory distress syndrome [33]. In myocardial infarction and myocarditis, emodin exerts its protective effects also through the regulation of inflammation $[12,13]$. In the present study, we focused on the influences of emodin on the concentration of proinflammatory cytokines IL- 6 and TNF- $\alpha$, and found that the increased inflammation induced by CHF was obviously suppressed by the treatment of emodin. It is implied that emodin might improve $\mathrm{CHF}$ by regulation of inflammation.

MiRNAs are a group of small noncoding RNAs and play crucial roles in various diseases, including cardiovascular diseases [34]. The clinical application of aberrant expression of key molecules especially for miRNAs has attracted increasing attention in disease treatment $[35,36]$. In recent years, the role of miRNAs acted in the mechanisms of the protective effects of emodin has been demonstrated. For instance, emodin was demonstrated to attenuate myocarditis via downregulation of miR-223 [14]. MiR-138 was proved to mediate the protective effects of emodin against myocardial infarction [15]. In pancreatic cancer, emodin was found to inhibit the cancer cell EMT and invasion 
by upregulating the expression of miR-1271 [37]. In our study, we observed a markedly decrease in the expression of miR-26b-5p in CHF rats compared with the controls. More importantly, the reduced miR-26b-5p in the CHF model was promoted by the treatment of emodin. Additionally, the cardiac function of the $\mathrm{CHF}$ rats was remarkably improved by the upregulation of miR-26b-5p, and the improved cardiac function induced by emodin was abrogated by the downregulation of miR-26b-5p, which implied that miR-26b-5p might be involved in the development of $\mathrm{CHF}$ and participate the effects of emodin.

Previous studies have reported that miR-26b-5p was implicated in inflammation [38]. In our study, the inflammatory response was improved by the overexpression of miR-26b-5p evidenced by the reduced IL- 6 and TNF- $\alpha$ concentration. Furthermore, we found that the reduced levels of inflammatory cytokines attributed to emodin treatment were rescued by the knockdown of miR-26b-5p, indicating that emodin improved the inflammation of CHF rats through upregulating miR-26b-5p. To further explore the molecular mechanisms of emodin in CHF treatment, the target gene of miR26b-5p was predicated and PTEN as a potential target was demonstrated in this study. PTEN is an upstream regulator of the PI3K/AKT signaling, and this signaling has been reported to mediate the protective effects of Qishenkeli against myocardial apoptosis in CHF $[39,40]$. Thus, we suspected that the emodin might improve the cardiac function and inflammation by regulation of miR-26b$5 p$ and the downstream PTEN/PI3K/AKT signaling pathway in CHF rats. This hypothesis needs to be verified in further studies.

In conclusion, all the data in this study revealed that emodin plays a protective role against the impaired cardiac function and dysregulation in inflammation in CHF rats, and its effects may be achieved by regulating the miR-26b-5p/PTEN pathway. Our discovery indicates that emodin has an obviously potential as an anti-CHF therapeutic agent.

\section{Conflicts of interest}

The authors declare no conflict of interest.

\section{References}

1. Aune D, Keum N, Giovannucci E, et al. Nut consumption and risk of cardiovascular disease, total cancer, allcause and cause-specific mortality: a systematic review and dose-response meta-analysis of prospective studies. BMC Med 2016; 14: 207.

2. Brake R, Jones ID. Chronic heart failure part 2: treatment and management. Nurs Stand 2017; 31: 53-63.

3. Hoffman TM. Chronic heart failure. Pediatr Crit Care Med 2016; 17: S119-23.
4. Maggioni AP. Epidemiology of heart failure in Europe. Heart Fail Clin 2015; 11: 625-35.

5. Khatibzadeh S, Farzadfar F, Oliver J, Ezzati M, Moran A. Worldwide risk factors for heart failure: a systematic review and pooled analysis. Int J Cardiol 2013; 168: 1186-94.

6. Yoshimura M, Yasue H, Ogawa H. Pathophysiological significance and clinical application of ANP and BNP in patients with heart failure. Can J Physiol Pharmacol 2001; 79: 730-5.

7. Kaplan P, Vrtovec B, Jug B. Orosomucoid is an independent predictor of prognosis in chronic heart failure. Wien Klin Wochenschr 2016; 128: 870-4.

8. Dong $X$, Fu J, Yin X, et al. Emodin: a review of its pharmacology, toxicity and pharmacokinetics. Phytother Res 2016; 30: 1207-18.

9. Chen H, Huang RS, Yu XX, et al. Emodin protects against oxidative stress and apoptosis in HK-2 renal tubular epithelial cells after hypoxia/reoxygenation. Exp Ther Med 2017; 14: 447-52.

10. Zhang W, Lu X, Wang W, et al. Inhibitory effects of emodin, thymol, and astragalin on leptospira interrogans-induced inflammatory response in the uterine and endometrium epithelial cells of mice. Inflammation 2017; 40: 666-75.

11. Sharma R, Tiku AB. Emodin inhibits splenocyte proliferation and inflammation by modulating cytokine responses in a mouse model system. J Immunotoxicol 2016; 13: 20-6.

12. Song ZC, Wang ZS, Bai JH, Li Z, Hu J. Emodin, a naturally occurring anthraquinone, ameliorates experimental autoimmune myocarditis in rats. Tohoku J Exp Med 2012; 227: 225-30.

13. Wu Y, Tu X, Lin G, et al. Emodin-mediated protection from acute myocardial infarction via inhibition of inflammation and apoptosis in local ischemic myocardium. Life Sci 2007; 81: 1332-8.

14. Yang Y, Jiang Z, Zhuge D. Emodin attenuates lipopolysaccharide-induced injury via down-regulation of miR-223 in H9c2 cells. Int Heart J 2019; 60: 436-43.

15. Zhang X, Qin Q, Dai H, Cai S, Zhou C, Guan J. Emodin protects $\mathrm{H} 9 \mathrm{c} 2$ cells from hypoxia-induced injury by up-regulating miR-138 expression. Braz I Med Biol Res 2019; 52: e7994.

16. Vegter EL, Ovchinnikova ES, van Veldhuisen DJ, et al. Low circulating microRNA levels in heart failure patients are associated with atherosclerotic disease and cardiovascular-related rehospitalizations. Clin Res Cardiol 2017; 106: 598-609.

17. Ge ZW, Zhu XL, Wang BC, et al. MicroRNA-26b relieves inflammatory response and myocardial remodeling of mice with myocardial infarction by suppression of MAPK pathway through binding to PTGS2. Int J Cardiol 2019; 280: 152-9.

18. Tveden-Nyborg P, Bergmann TK, Lykkesfeldt J. Basic and clinical pharmacology and toxicology policy for Experimental and Clinical studies. Basic Clin Pharmacol Toxicol 2018; 123:233-235

19. Wang EW, Jia XS, Ruan CW, Ge ZR. miR-487b mitigates chronic heart failure through inhibition of the IL-33/ST2 signaling pathway. Oncotarget 2017; 8: 51688-702.

20. Hao P, Jiang F, Cheng J, Ma L, Zhang Y, Zhao Y. Traditional Chinese medicine for cardiovascular disease: evidence and potential mechanisms. J Am Coll Cardiol 2017; 69: 2952-66.

21. Cao Y, Zhang PY. Review of recent advances in the management of hypertrophic cardiomyopathy. Eur Rev Med Pharmacol Sci 2017; 21: 5207-10. 
22. Xian SX, Yang ZQ, Ren PH, et al. Effect of yangxinkang tablets on chronic heart failure: a multi-center randomized double-blind placebo-controlled trial. Chin J Integr Med 2015; 21: 733-42.

23. Li X, Zhang J, Huang J, et al. A multicenter, randomized, double-blind, parallel-group, placebo-controlled study of the effects of qili qiangxin capsules in patients with chronic heart failure. J Am Coll Cardiol 2013; 62: 1065-72.

24. Pang X, Liu J, Li Y, Zhao J, Zhang X. Emodin inhibits homocysteine-induced $C$-reactive protein generation in vascular smooth muscle cells by regulating PPARgamma expression and ROS-ERK1/2/p38 signal pathway. PLoS One 2015; 10: e0131295.

25. Moreno-Eutimio MA, Espinosa-Monroy L, Orozco-Amaro T, et al. Enhanced healing and anti-inflammatory effects of a carbohydrate polymer with zinc oxide in patients with chronic venous leg ulcers: preliminary results. Arch Med Sci 2018; 14: 336-44.

26. Eken MK, Ersoy GS, Kaygusuz El, et al. Etanercept protects ovarian reserve against ischemia/reperfusion injury in a rat model. Arch Med Sci 2019; 15: 1104-12.

27. Trocha M, Merwid-Lad A, Piesniewska M, et al. Age-related differences in function and structure of rat livers subjected to ischemia/reperfusion. Arch Med Sci 2018; 14: 388-95.

28. Lam HB, Yang PS, Chien MN, Lee JJ, Chao LF, Cheng SP. Association between neutrophil-to-lymphocyte ratio and parathyroid hormone in patients with primary hyperparathyroidism. Arch Med Sci 2019; 15: 880-6.

29. Prabhu SD, Frangogiannis NG. The biological basis for cardiac repair after myocardial infarction: from inflammation to fibrosis. Circ Res 2016; 119: 91-112.

30. Cooper LT Jr. The changing face of cardiac inflammation: new opportunities in the management of myocarditis. Circ Heart Fail 2017; 10. DOI: 10.1161/CIRCHEARTFAILURE.117.004528.

31. Shirazi LF, Bissett J, Romeo F, Mehta JL. Role of inflammation in heart failure. Curr Atheroscler Rep 2017; 19: 27.

32. Chen YK, Xu YK, Zhang $H$, et al. Emodin alleviates jeju num injury in rats with sepsis by inhibiting inflammation response. Biomed Pharmacother 2016; 84: 1001-7.

33. Zhu T, Zhang W, Feng SJ, Yu HP. Emodin suppresses LPS-induced inflammation in RAW264.7 cells through a PPARgamma-dependent pathway. Int Immunopharmacol 2016; 34: 16-24.

34. Ding S, Huang H, Xu Y, Zhu H, Zhong C. MiR-222 in cardiovascular diseases: physiology and pathology. Biomed Res Int 2017; 2017: 4962426.

35. Jedynak M, Siemiatkowski A, Milewski R, Mroczko B, Szmitkowski $M$. Diagnostic effectiveness of soluble triggering receptor expressed on myeloid cells-1 in sepsis, severe sepsis and septic shock. Arch Med Sci 2019; 15: 713-21.

36. Jesionek-Kupnicka D, Braun M, Trabska-Kluch B, et al. MiR-21, miR-34a, miR-125b, miR-181d and miR-648 levels inversely correlate with MGMT and TP53 expression in primary glioblastoma patients. Arch Med Sci 2019; 15: 504-12.

37. Li N, Wang C, Zhang P, You S. Emodin inhibits pancreatic cancer EMT and invasion by upregulating microRNA1271. Mol Med Rep 2018; 18: 3366-74.

38. Kang YC, Zhang L, Su Y, Li Y, Ren WL, Wei WS. MicroR$\mathrm{NA}-26 \mathrm{~b}$ regulates the microglial inflammatory response in hypoxia/ischemia and affects the development of vascular cognitive impairment. Front Cell Neurosci 2018; 12: 154

39. Perez-Ramirez C, Canadas-Garre M, Molina MA, FausDader MJ, Calleja-Hernandez MA. PTEN and PI3K/AKT in non-small-cell lung cancer. Pharmacogenomics 2015; 16: 1843-62.

40. Chang H, Li C, Wang Q, et al. OSKL protects against myocardial apoptosis on heart failure via PI3K/Akt-p53 signaling pathway. Sci Rep 2017; 7: 16986. 